\title{
An inquiry into the morphology of Ciliate Protozoa using an engineering design approach
}

\author{
E. L. Benjamin ${ }^{1}$, M. W. Collins ${ }^{2}$ \& D. McL. Roberts ${ }^{3}$ \\ ${ }^{1}$ Faculty of Business, Computing \& Information Management, \\ London South Bank University, London, UK \\ ${ }^{2}$ School of Engineering and Design, Brunel University, London, UK \\ ${ }^{3}$ Department of Zoology, The Natural History Museum, London, UK
}

\begin{abstract}
Although cellular morphogenesis is an area of microbiology that is widely studied, very little is understood about the manner in which cells shape themselves or how they optimise their form to their environment. There exists an extensive literature on body shape, anatomy and life cycle of numerous single-celled micro-organisms including both prokaryotes and eukaryotes. However, with regards to morphology itself, there is a shortage of general empirical relationships to enable the interknitting of specific features that could lead to a biologically justifiable generic form. This paper comprises a concise review of the subject together with an assessment of the experimental approach which could be used, within an intended overall context of Engineering Design. Keywords: morphology, morphogenesis, prokaryotes, eukaryotes, confocal microscope, microtubule, kineties, buccal cavity, oral apparatus, cytoskeleton, protozoa, ciliates, flagellates, protists.
\end{abstract}

\section{Introduction}

The cell shape is itself a characteristic property of a species. It would be most advantageous if the parameters of the surface could be used directly as characters themselves, particularly if their values could be used as a vector to define a location in some (morpho-) space, and with a distance between such locations being a measure of the difference between individuals. Sets of such vectors could then be subjected to cluster analysis and other grouping techniques to 
uncover or establish relationships. The fitting of the extracted surface to a generic model is an application of several established techniques [21-26].

\subsection{Biology}

A fundamental objective of classical morphology in microbiology is the identification and classification of the basic shapes that cells can assume; to display morphological regularities corresponding to familiar categories, and, if possible to express mathematically the harmony of forms. This approach was proposed by Harold [1] but because it was not attractive to microbiologists, he did not pursue it. Instead however, he attempted to formulate 'casual' principles to explain how biological forms arise and how they are transmitted from parent to offspring. This approach has problems, as Harold appreciated, because microbial forms are so diverse that there is no obvious unitary principle of morphogenesis, in the sense that unitary principles do account for heredity, protein synthesis, or oxidative phosphorylation. On the other hand, it seemed implausible to him that each organism is altogether unique. Harold therefore suggested that the greater likelihood is that the multitudinous forms represent variations on a much smaller number of generative themes, whose discovery is the proper goal of research in cellular topobiology.

While Harold's review is relatively recent - 1990, he harks back to the classic approach of D'Arcy Thompson, On Growth and form, first published in 1917. In Bonner's Editorial Introduction to the Canto abridged reprint [2], he focuses on D'Arcy Thompson's “... most conspicuous attitude [was] the analysis of biological processes from their mathematical and physical aspects" (pp. xiv). Specifically, his analyses related biological forms, and their changes "apparent in ... movements and ... growth ....as due to the action of force", namely "a diagram of forces" ([2], pp. 11).

This approach has an enduring appeal, not least in Ball's rather beautiful The Self-Made Tapestry [3]. D'Arcy Thompson features throughout ([3] pp. 1 and pp. 251-253, for example) and Ball concludes that whereas D'Arcy Thompson "was unable to persuade most of his peers of the importance of form and pattern", now "pattern formation" (at least) is an "identifiable field of study in its own right."

For protozoa, the most significant current expression of D'Arcy Thompson's 'mathematical aspects' is that of Goodwin [4]. Applying various forms of Laplace's field equation to Tetrahymena (See figure 4) in particular, he was able to generate convincing mathematical models of the buccal cavity asymmetry and the ciliature ([4], pp. 338). A brief description of the buccal cavity is given in section 3.1 .

It is worthy to note Thompson's recognition that, "Many a beautiful protozoan form has lent itself to easy physico-mathematical explanation; others, no less simple and no more beautiful, prove harder to explain.” ([2], pp. 171]). 


\subsection{Rationale}

Even in the context of a single Tetrahymena, Goodwin noted that the "overall shape varies greatly, from pear to egg to cucumber" ([4], pp. 381). Figure 1 shows how the shape of protozoa varies from species to species.

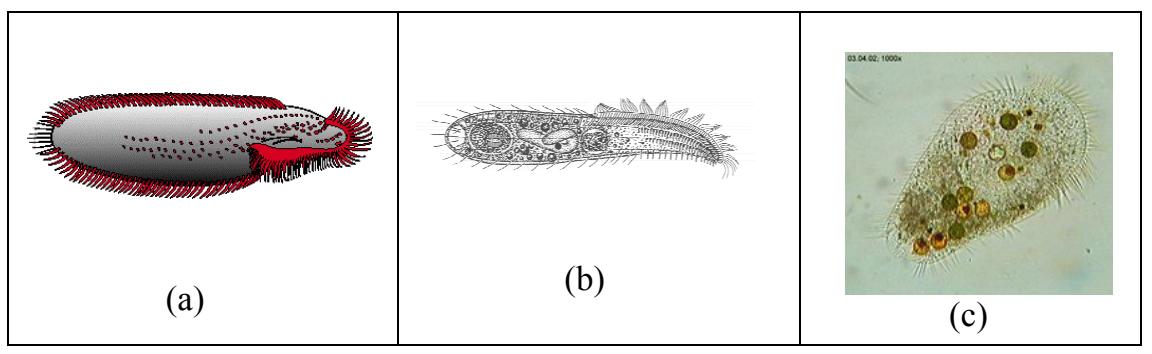

Figure 1: $\quad$ Some typical protozoa. (a) Hypotrichida (diagram from P. Eigner). (b) Type species: Cultellothrix velhoi sp. n. (diagram from Wilhelm Foissner). (c) Stichotrich. (from GNU Free Documentation License: http://en.wikipedia.org/wiki/Ciliate).

Bonner points out that D'Arcy Thompson "was in no way an experimenter," ([2], pp. Xv), and it is very clear that actual experimental data would not be helpful in testing the approach described briefly before.

Our hypothesis is that ciliate cell surfaces can be described with a few rules and a few parameters. To test the hypothesis, we are seeking to assemble a dataset of confocal images of stained single cells, which will then be analysed, from an engineering and design perspective. In more detail 3-D CAD (Computer Aided Design) models will be used to describe a surface onto which the morphology of a ciliate cell can be mapped. If the results of this study are positive, it will mean that a small set of invariants will suffice to describe the cells and become an aid to automating the identification of the species themselves and of quantifying the effects of variation due to environmental influences.

\section{Mathematical modelling}

\subsection{D'Arcy Thompson's legacy}

To an intriguing extent, the foundation of D'Arcy Thompson's explanations is that of engineering, as so aptly reiterated by Harold [1].

"The objects that surround us in daily life, most of them man-made, were produced by shaping a natural or artificial material to suit some function or purpose. They owe their form to the application of physical forces, most commonly mechanical ones. The idea that biological forms likewise reflect the action of physical forces on formless protoplasm is a venerable one, although somewhat unfashionable today." ([1], pp. 393). 
The first section of D'Arcy Thompson's Chapter II 'The principle of similitude' ([2], pp. 15) corresponds to sections relating to convective heat transfer in a typical UK Undergraduate text on Thermodynamics - "The principles of dynamic similarity and dimensional analysis applied to forced convection' ([5], pp. 571), for example.

\subsubsection{Cartesian transformations}

The above correspondence with engineering goes further. D'Arcy Thompson described "a net of rectangular equidistant co-ordinates (about the axes of $\mathrm{x}$ and y)," and showed that by altering or deforming the "network in various ways," an organism's appearance can be modified to resemble different species. This is predicated on the assumption that the organisms display a reasonable number of homologous (directly equivalent) landmark features and some smooth function exists to determine their spatial relationship.

Thompson stated "It follows that any figure which we may have inscribed in the original net, and which we transfer to the new, will thereby be deformed in strict proportion to the deformation of the entire configuration, being still defined by corresponding points in the network and being throughout in conformity with the original figure. For instance, a circle inscribed in the original 'Cartesian' net will now, after extension of the y-direction, be found elongated into an ellipse." ([2], pp. 276).

Figure 2 illustrates Thompson's approach.

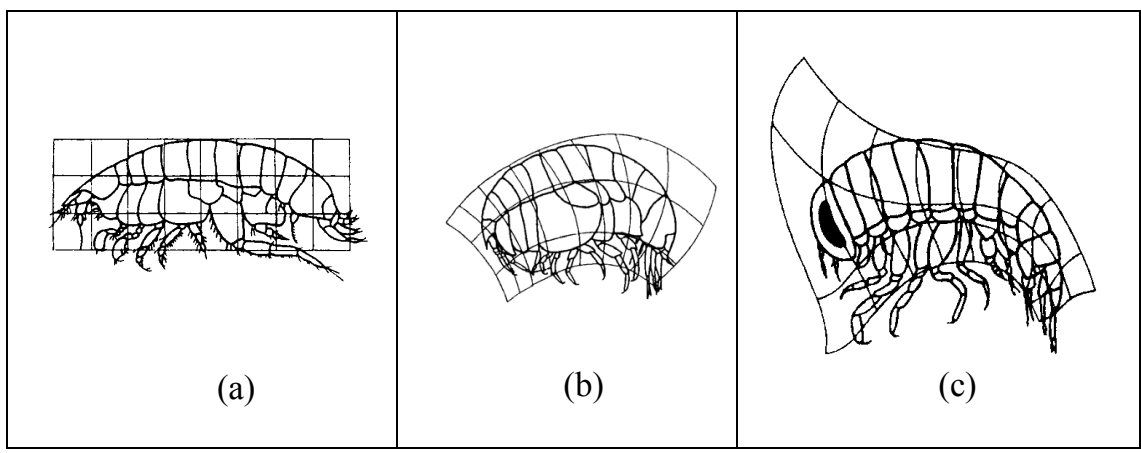

Figure 2: (a) Harpinia plumose $\mathrm{Kr}$. a little amphoid of the family Phoxocephalidae (Harpinia sp.) (b) By deforming the co-ordinates of Harpinia into the curved orthogonal system, we at once obtain a very fair representation of the allied genus, belonging to a different family of amphoids, namely Stegocepahalus (c) Greater deformation of Harpinia or Stegocephalus results in a tolerable representation of the aberrant genus Hyperia, with its narrow abdomen, its reduced pleural lappets, its great eyes, and its inflated head. (From D’Arcy Thompson, with his explanation, [2], pp. 295).

Now D'Arcy Thompson's 'similitude' and 'method of coordinates' may be regarded as subsumed within the relatively recent engineering developments of 
Computational Fluid Dynamics (CFD) and heat transfer - that is a modelling of the basic non-linear partial differential equations in preference to the prior 'dimensional analysis.'

At one stage, the CFD griding techniques involved co-ordinate transformation, of, if needed, only a part of the field, so in a CFD simulation of flying or swimming an overall Cartesian grid, could include local co-ordinate systems as in Figure 2. For 'solid' locomotion, outside CFD, the same principles could be applied.

What it means is that efficient alternative 'locomotive' shapes are possible, where simple coordinate transformation rules apply.

\subsection{Preston's legacy}

In 1953, F. W. Preston [6] undertook studies on the shape of birds' eggs. "This investigation was not undertaken primarily as a mathematical amusement. It seems likely that it may throw some light on several biological and ecological problems, but the present paper concerns itself namely with the broad question of what is the shape of a bird's egg. The mathematics may conceivably show something of the physiology and mechanics of egg-laying, since the shape of the egg is a response to the forces exerted by the oviduct during shell-formation (Mallock, 1925; D’Arcy Thompson, 1943)." ([7], pp. 160). Figure 3 shows Preston's circles of derivation.

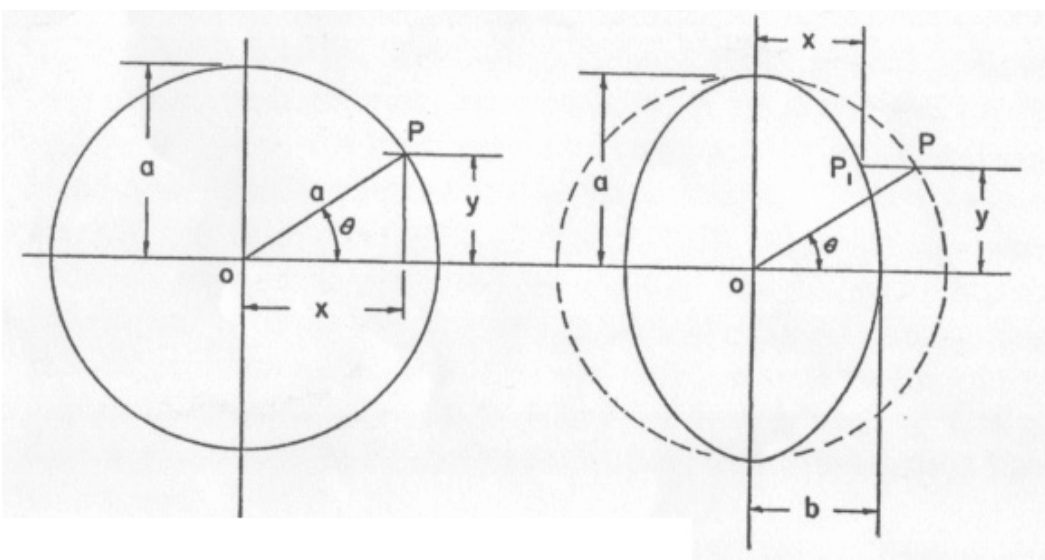

Figure 3: (left) Parametric equation of a circle in terms of eccentric angle. (right) Parametric equation of an ellipse. (From Preston with this explanation [6], pp. 161).

Starting with the parametric equation of a circle, Preston described the representation of birds' eggs in terms of 'circles of derivation', which then describes the various shapes.

$$
y=a \operatorname{Sin} \theta ; \quad x=b \operatorname{Cos} \theta\left(1+c_{1} \operatorname{Sin} \theta+c_{2} \operatorname{Sin}^{2} \theta+c_{3} \operatorname{Sin}^{2} \theta\right)
$$


which, except in the case of the Alcids, reduces to:

$$
y=\operatorname{Sin} \theta ; \quad x=b \operatorname{Cos} \theta\left(1+c_{1} \operatorname{Sin} \theta+c_{2} \operatorname{Sin}^{2} \theta\right)
$$

where $\mathrm{c}$ is a dimensionless parameter that may vary from egg to egg but is constant for any particular specimen.

for a few eggs: $y=\operatorname{Sin} \theta ; \quad x=b \operatorname{Cos} \theta\left(1+c_{1} \operatorname{Sin} \theta\right)$

and for a few others, whose ends are virtually alike, we may have:

$$
y=\operatorname{Sin} \theta ; \quad x=b \operatorname{Cos} \theta\left(1+c_{2} \operatorname{Sin}^{2} \theta\right) .
$$

Preston's work was supported by Okabe (“On the Form of Hens' eggs" Reports of Research Institute for Applied Mechanics, Kyushu University, Vol. 1, 1952). Okabe expressed the view that Preston's treatment of the shape of eggs, "as a problem in the forces that mould that shape," and then using the shape to interpret the interacting forces, is in tune with the desires of D'Arcy Thompson [6, pp. 161].

However, it should be appreciated that the model is an arbitrary geometric simulation, so should not be used predictively outside its range.

Very recently, Narushin [7, 8] developed further the following mathematical equation for bird's eggs profile.

$$
\mathrm{Y}= \pm \sqrt{ }\left(\mathrm{L}^{2 /(\mathrm{n}+1)} \mathrm{x}^{2 \mathrm{n} /(\mathrm{n}+1)}-\mathrm{x}^{2}\right) \quad \text { in which } \mathrm{n}=1.057(\mathrm{~L} / \mathrm{B})^{2.372}
$$

where $\mathrm{L}$ the length of the egg, $\mathrm{B}$ the maximum breadth of the egg, $\mathrm{X}$ the coordinate along the longitudinal axis and $\mathrm{y}$ is the traverse distance to the profile. Naurashin has proposed "Indices of ellipticity and conicity for the calculation of the deviation of an egg from its theoretical profile, defined by the above equation..." On the same basis, "...formulae for the calculation of an egg's volume, surface area, longitudinal circumference length and normal projected area..." have also been proposed [8]. However, these derivations have been omitted here in the interest of space.

\subsection{Goodwin's proposition}

Goodwin's approach has already been mentioned. Space does not allow a full description of this but figure 4 shows the outcome of progressive developments in the Laplace equation.

Goodwin described the morphological features of Tetrahymena (figure 4(a)) by using the spherical shape of the organism as the basic shape, and using the basic polar organisation of its cortical field, to determine the global spatial ordering of the cilia. Figure 4(b) shows the anterior and posterior as north $(\mathrm{N})$ and south $(\mathrm{S})$ poles respectively. Goodwin further described this polarising field with the following general equation:

$$
\mathrm{U}(\theta, \varphi)=\alpha \ln \operatorname{Cot}(\theta / 2)+\beta \varphi-\mathrm{b} \operatorname{Cot}(\theta / 2) \operatorname{Cos} \varphi
$$


in which $\theta$ and $\varphi$ determine the two locations, latitude and longitude respectively, $\alpha$ a parameter that determines the strength of the field, and $U_{0}$ a function which defines the poles. It varies from $\theta=\pi(-\infty)$ at the posterior pole to $\theta=0(+\infty)$ at the anterior pole. $\beta$ is a parameter which determines the spiral orientation of the cilia. A negative value of $\beta$ indicates a clockwise spiral and a positive value indicates an anticlockwise spiral. $\beta=0$ is indicative of the rare case of a non-spiral polarity.

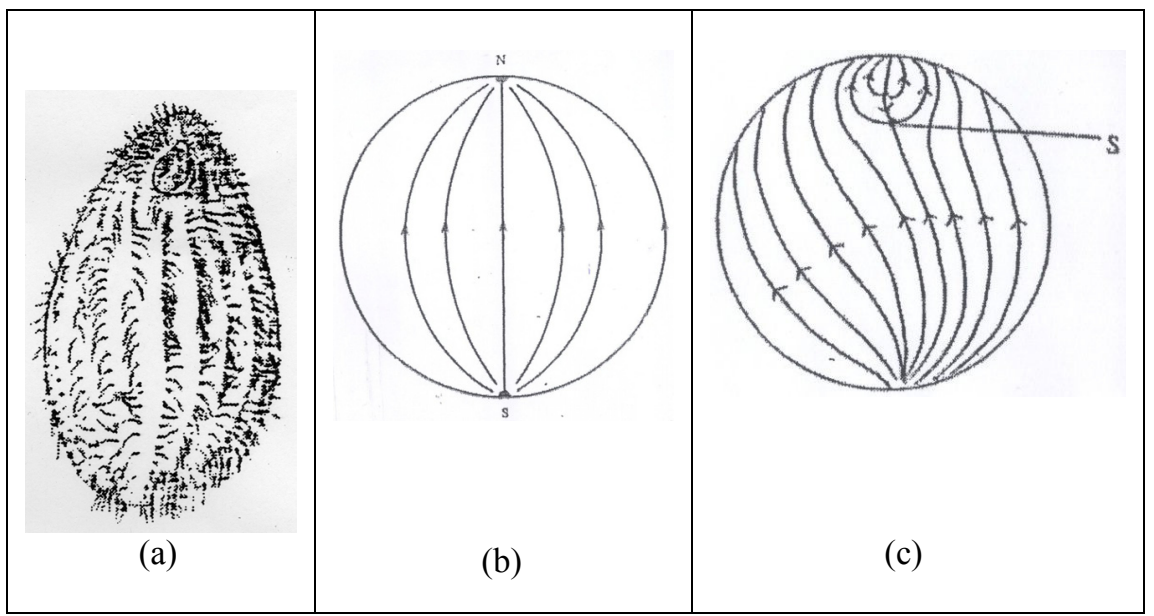

Figure 4: (a) Tetrahymena pyriformis, showing ciliary meridians and buccal cavity with its ciliture (See section 3.1 below). (b) ploarised meridians on the sphere defined by the basic polar field. (c) Members of the two families of curves on the sphere of equation (1) and (2). The arrows define the direction of increasing $\mathrm{U}(\theta, \varphi) \mathrm{S}$, which established posterior-anterior polarity saddle points. (From Goodwin, with his explanation, [4], pp. 381, 383 and 388).

The parameter $b$ takes into account the presence of the bucal cavity, a 'saddle point' depicting loss of polarity with respect to a Laplacean field. It is the measure or strength of asymmetry about the polar axis. For organisms lacking the asymmetry such as that of the ciliature spiral pattern found in Tetrahymena pyriformis, for example, $\mathrm{b}=0$. Further analysis gives rise to the equation:

$$
\mathrm{V}(\theta, \varphi)=+\beta \ln \operatorname{Cot}(\theta / 2)-\alpha \varphi+\mathrm{b} \operatorname{Cot}(\theta / 2) \operatorname{Sin} \varphi
$$

in which $\mathrm{V}(\theta, \varphi)$ is a harmonic conjugate function of $\mathrm{U}(\theta, \varphi)$ and describes the longitudinal direction which is orthogonal to the cilia meridians. Figure 4(c) shows curves corresponding to the kineties (ciliary meridians) in T. pyriformis, defined by $\mathrm{V}(\theta, \varphi)=$ constant. As iterated by Goodwin, each curve corresponds 
to a different value of the constant. Note that "The field geometry ... implies that the oral apparatus and the ciliary meridians are spatially organised by the same global field." [4]. This is reiterated by Webster and Goodwin in their writing on Morphometric fields and cortical inheritance [28]. Another narrative worthy of note, is that of Goodwin's on A Generative Biology: Morphometric fields [27].

\section{Protists}

We now review the evolutionary history of the protists.

Single eukaryotic cells, the Protista, evolved at some distant time in the evolutionary past, probably more than 2,000 Myr ago. Prokaryotic life almost certainly evolved very soon after there was liquid water available, more than 4,000 Myr ago. Eukaryotes differ from prokaryotes in many fundamental ways relating to cell structure and organisation. Probably most importantly, they posses a cytoskeleton which acts both as a frame against which forces generated by flagella can act (see section 3.2) and which permits the capture of particulate food.

By contrast, the earliest evidence for multicellular life does not appear until around $800 \mathrm{Myr}$ ago, so the major chemical cycles evolved in an unicellular context. Multicellular organisms evolved in the presence of a fully-functional ecosystem.

\subsection{Ciliated protists}

Ciliated protozoa are one of the very few traditional groups of protists which are believed to be a good group in the context of modern evolutionary understanding. They are the best-studied of the non-pathogenic protists and have an established place in the laboratory where they are fairly easily manipulated [13]. They are large enough to image easily with light microscopy and are amenable to routine cytological staining $[13,18,19,20]$. Staining of surface features for confocal microscopy is a known art, and a functional method was published by Ann Fleury [14, 15], Fleury and Laurent [6] and Fleury et al. [17].

Ciliates are supported by a microtubular cytoskeleton which runs from anterior to posterior pole. See figure 4(a). Somatic (body) cilia, responsible for locomotion, are arranged in rows (meridians) called kineties which also run from pole to pole $[11,12,29 \mathrm{pp} .6-7]$. The cell's mouth can be at the anterior pole or, more commonly, on the ventral surface. In transverse section they can be round or somewhat compressed (oblate). The shapes can be readily approximated by an asymmetric spheroid. The asymmetry required means that the widest point on the major axis is not necessarily located in the middle of the cell, for instance a pear-shape. Such shapes can be easily constructed by empirical equations with minimal parameterization (for example, see section 2.2 above), but there is no known theoretical reason yet established to choose one equation form over another. 
The body characters, such as the kineties, are organised in a variety of ways. It is not known whether changes to the body shape affect the organisation beyond certain dramatic changes, such as the increase in the density of kineties in response to starvation condition to produce swarmer cells, adapted to swim faster and believed to be a dispersal mechanism.

The organisation of the oral (mouth) cilia is a central feature of ciliate taxonomy. In general the mouth is located at the bottom of a depression in the body called a vestibulum or buccal cavity. The oral cilia are organised into arrays called membranelles, which are designed to funnel water towards the mouth where particulate food is trapped by the final membranelle. The fluid dynamics of this process have been briefly studied by Tom Frenchel [9].

\subsection{Flagellated protists}

Flagellated protists are generally smaller than ciliates and most commonly carry two flagella (singular, flagellum) of unequal length. Flagella and cilia have an identical structure, although flagella tend to be longer and cells bear fewer of them, the different terms are maintained in the community for historical consistency. Cilia beat with an oar-like stroke [10] while the longer flagella have a sinusoidal stroke and, with a whip-like action ([29], pp. 11-13), normally move water from tip to base, so pulling the cell through the water. Some flagellates (the Stramenopiles) possess tripartite hair-like projections called retronemes along the length of the flagellum. As their name suggests, the effect of the retronemes is to reverse the flow of water as it travels from base to tip, so pushing the cell through the water.

Morphologically, flagellates are far more diverse than ciliates and it is believed that the ancestral eukaryotes carried a flagellum. They can also be phagotrophic, although osmotrophic and phototrophic lifestyles are also widespread. There are correspondingly many ways that cells capture particulate prey, including a gullet, tentacles which may act as a filter and a variety of adhesive mechanisms.

\section{Conclusions}

This research has three main themes of interest. It is strongly interdisciplinary, being a combination of the latest biological research interest allied with an engineering design approach. Secondly, it has a strong computational science focus and it is possible that subject to successful demonstration, the methods could apply more widely in morphogenesis. Finally, in combination with addressing the fluid dynamics of the nutrient dependent ciliate shape, it is intended to explore the water cleansing effectiveness of protists, leading to improved biological treatment of sewage systems. The research programme is therefore anticipated as contributing to three principle areas.

First, the academic question of evolutionary relationships and the problem of grouping organisms into informative assemblages. Unicellular eukaryotes are now understood to be an exceedingly diverse collection of taxa which have 
traditionally, but erroneously, been classified by their morphological and functional similarity (e.g. the algae or the amoebae). We now recognise that these groups are intimately mixed in evolutionary descent based largely on discoveries made through molecular biology. Unfortunately, few taxa have been studied with molecular techniques or by detailed electron microscopy. Those that have are not well distributed amongst the recognized diversity of the group. Increased knowledge of body-plan and cellular organisation, based on a functional understanding, will contribute to efforts to integrate the classification of described taxa, particularly those that might be well described as legacy data.

Second, the organisation of characters on the body is known to change in response to environmental condition. Current methods can only detect gross changes in morphology. An improved method of assessing body conformation, specifically separating size and shape from component organisation, may well prove to be a powerful tool for monitoring environmental change. Such an approach is applicable in two domains: first, academically in ecology, to assign an individual or population to an ecological role. Second, practically, to provide a rapid-response method of assessing water quality.

Third, there is the practical question of how protists manage to remove bacteria from water so efficiently. The removal of very small particles (of the order of unit microns) from water is dominated by viscous forces. Such removal is industrially very important both in public health (the removal of pathogens from water) and in industry, for clean technologies. A better understanding of how protists achieve their filtration, which can even be selective, could lead to novel engineering solutions to this problem.

\section{References}

[1] Harold, F. M., To shape a cell: an inquiry into the causes of morphogenesis of microorganisms. Microbiological Reviews, 54(4), pp. 381-431, 1990.

[2] Thompson, D. W., On growth and form. Abridged, J. T. Bonner (ed). Cambridge University Press, London, 1961.

[3] Ball, P., The self-made tapestry: Pattern formation in nature. Oxford University Press, 1999.

[4] Goodwin, B. C., Pattern formation and its regeneration in the protozoa. Sym. Soc. Gen. Microbiology, 30, pp. 377-404, 1980.

[5] Rogers, G. F. C. \& Mayhew, Y., Engineering Thermodynamics, Work and heat Transfer, 4th ed., Prentice Hall, Harlow, UK.

[6] Preston, F. W., The shape of bird's eggs. The Auk, 70, pp. 160-182, 1953.

[7] Narushin, V. G., The avian egg: geometrical description and calculation of parameters. J. Agric. Engng. Res., 68, pp. 201-205, 1997.

[8] Narushin, V. G., Shape geometry of the avian egg. J. Agric. Engng. Res., 79, pp. 441-448, 2001.

[9] Fenchel, T., Protozoan filter feeding. Progress in Protistology, 1, pp. 65-113, 1986.

[10] Sleigh, M. A., The Biology of Protozoa, London: Edward Arnold, 1973. 
[11] Grell G. G., Protozoology. Springer-Verlag: Berlin, Heidelberg and New York, 1973.

[12] Corliss, J. O., The ciliated Protozoa: Characterization, classification, and guide to the literature. Pergamon Press: Oxford, London, New York and Paris, 1961.

[13] Roberts, D. McL. \& Causton, H., Silver nitrate impregnation of ciliated protozoa. Arch. Protistenkd. 135, pp. 299-318, 1998.

[14] Fleury, A., Dynamics of the cytoskeleton during morphogenesis in the ciliate Euplotes. 1. Basal bodies related microtubular system. Europ. J. Protistol., 27(2), pp. 99-114, 1991.

[15] Fleury, A., Dynamics of the cytoskeleton during morphogenesis in the ciliate Euplotes. 2. Cortex and continuous micro tubularsystems. Europ. J. Protistol., 27(3), pp. 220-237, 1991.

[16] Fleury, A., \& Laurent, M., Transmission of surface pattern through a dedifferentiated stage in the ciliate Paraurostyla. Evidence from the analysis of the microtubule and Basal body development. J. Euk. Microbiol., 41(3), pp. 276-291, 1994.

[17] Fleury, A., Le Guyader, H., Iftode, F., Laurent, M. \& Bornens, M., A scaffold for basal body patterning revealed by a mono-clonal antibody in the Hypotrich Ciliate Paraurostyla weissei. Dev. Biol., 157, pp. 285-302.

[18] Garreau de Loubresse, N., Keryer G., Migues, B. \& Beisson, J., A contractile cytoskeleton network of Paramecium: the infracilliary lattice. J. Cell Sci., 90, pp. 351-364.

[19] Silva-Neto, I. D. da, Improvement of silver impregnation technique (protargol) to obtain Morphological features of protists ciliates, flagellates and opalinates. Rev. Brasil. Biol., 60(3), pp. 451-459, 2000.

[20] Nicolina, D., Renato, A., Mortara, N. L., Morphological and physiological changes in Tetrahymena pyriformis for the in Vitro cytotoxicity assessment of Triton X-100. Toxicology in Vitro, 17, pp. 357-366, 2003.

[21] Alt, W., Statistics and Dynamics of cellular shape changes (Chapter 15). On Growth and Form: Spatio-temporal pattern formation in Biology, ed. M. A. J. Chaplain, G. D. Singh \& J. C. McLachlan, Wiley, UK, pp. 288-307, 1999.

[22] Auffray, J., Debat, V. \& Alibert, P., Shape asymmetry and developmental stability (Chapter 16). On Growth and Form: Spatio-temporal pattern formation in Biology, ed. M. A. J. Chaplain, G. D. Singh \& J. C. McLachlan, Wiley, UK, pp. 309-324, 1999.

[23] Lele, S., Invariance and Morphometrics: A critical appraisal of statistical techniques for landmark data (Chapter 17). On Growth and Form: Spatiotemporal pattern formation in Biology, ed. M. A. J. Chaplain, G. D. Singh \& J. C. McLachlan, Wiley, UK, pp. 325-336, 1999.

[24] Mardia, V., Statistical shape analysis and its applications (Chapter 18). On Growth and Form: Spatio-temporal pattern formation in Biology, ed. M. A. J. Chaplain, G. D. Singh \& J. C. McLachlan, Wiley, UK, pp. 337-355, 1999. 
34 Design and Nature III: Comparing Design in Nature with Science and Engineering

[25] Lozanoff, S., Sphenoethmoidal Growth, malgrowth and midfacial profile (Chapter 19). On Growth and Form: Spatio-temporal pattern formation in Biology, ed. M. A. J. Chaplain, G. D. Singh \& J. C. McLachlan, Wiley, UK, pp. 357-372, 1999.

[26] O’Higgins, P., Ontogeny and Phylogeny: Some morphometric approaches to skeletal growth and evolution (Chapter 20). On Growth and Form: Spatio-temporal pattern formation in Biology, ed. M. A. J. Chaplain, G. D. Singh \& J. C. McLachlan, Wiley, UK, pp. 373-393, 1999.

[27] Goodwin, B. C., D'Arcy Thompson and the problem of biological form (Chapter 21). On Growth and Form: Spatio-temporal pattern formation in Biology, ed. M. A. J. Chaplain, G. D. Singh \& J. C. McLachlan, Wiley, UK, pp. 395-402, 1999.

[28] Webster, G., \& Goodwin, B. C., Unitary morphogenetic field. Form and transformation, Cambridge university press, USA, pp. 221-229, 1996.

[29] Goodwin, B., C., How the leopard changed its spots, Princeton University Press, Princeton, New Jersey. 